\title{
An Incentive-Based Fairness Mechanism for Multi-Hop Wireless Backhaul Networks with Selfish Nodes
}

\author{
JengFarn Lee, Member, IEEE, Wanjiun Liao, and Meng Chang Chen, Senior Member, IEEE
}

\begin{abstract}
In this paper, we study the fairness problem in multi-hop wireless backhaul networks in the presence of selfish Transit Access Points (TAPs). We design an incentive-based mechanism which encourages TAPs to forward data for other TAPs, and thus eliminates the location-dependent unfairness problem in the backhaul network. We prove the correctness and truthfulness of the proposed mechanism, and evaluate its performance via ns-2 simulations. The results show that the proposed mechanism achieves fairness even when there are idle TAPs in the network.
\end{abstract}

Index Terms-Fairness, game theory, selfish node, wireless backhaul networks.

\section{INTRODUCTION}

$I^{\prime}$ EEE 802.11-based wireless network is a promising wireless technology to access the Internet due to the characteristics of low cost, robustness, and ease of deployment. Recently, there have been many studies to extend it from the traditional one-hop network access [1-4] to multi-hop communications, such as ad hoc networks, mesh networks, backhaul networks, etc. [5-8]. In a wireless backhaul network, traffic originating from or destined to mobile users is forwarded through multiple wireless Transit Access Points (TAPs) to or from the wired Internet via a gateway. However, under the Distributed Coordination Function (DCF) in 802.11, users located more hops away from the gateway suffer low throughput, and in some cases, even starvation. This calls for solutions to providing fair resource sharing for wireless backhaul networks.

In most existing work, all the nodes that make up a multihop network are assumed cooperative. In other words, they share a common goal, do not misbehave in packet forwarding, and are willing to act as a relay to forward data for the other nodes. This assumption, while reasonable in disaster recovery or military applications, may not hold for civilian backhaul networks, in which TAPs may be owned by different independent entities, such as restaurants, small business offices,

Manuscript received August 25, 2006; revised December 6, 2006; accepted February 2, 2007. The associate editor coordinating the review of this paper and approving it for publication was Q. Zhang.

J.F. Lee was with the Department of Electrical Engineering, National Taiwan University, Taipei, Taiwan, and is now with the Department of Computer Science and Information Engineering, National Chung-Cheng University, Chya-Yi, Taiwan (e-mail: jflee@cs.ccu.edu.tw).

W. Liao is with the Department of Electrical Engineering and the Graduate Institute of Communication Engineering, National Taiwan University, Taipei, Taiwan (e-mail: wjliao@cc.ee.ntu.edu.tw).

M. C. Chen is with the Institute of Information Science, Academia Sinica, Taipei, Taiwan (e-mail: mcc@iis.sinica.edu.tw).

Digital Object Identifier 10.1109/TWC.2008.060631. individual residences, or hot spots. Since TAPs are owned by profit-oriented independent agents, rather than a single entity, they are indeed selfish. That is to say, they will forward packets for other nodes only when the profit they make exceeds the cost they spend in forwarding. The selfish behavior of TAPs may result in low end-to-end throughputs for those TAPs located more hops away from the gateway, and worse, may even lead to service disruption.

The problem caused by nodes' selfish behavior has been recognized by the mobile ad hoc network community, and several mechanisms which encourage cooperation have been proposed [9]. Existing work may be either reputation-based (e.g., [10-14]), where the behavior of each node is monitored and non-cooperative nodes (including selfish and malicious ones) will be punished, or payment-based (e.g., [15-18]), where some virtual currency is introduced to encourage packet forwarding for other nodes. Other issues addressed in existing work, in addition to encouraging cooperation, include avoiding cheating [16][18] and collusion [15]. So far, very few efforts have been made to solve the fairness problem in wireless backhaul networks with selfish nodes. The challenges in that problem are mainly due to multi-hop relays, flow aggregation, and the underlying MAC layer mechanisms. In addition, the payment or revenue in backhaul networks comes mainly from mobile users, not TAPs or destinations as assumed in previous work. Thus, both reputation-based and payment-based mechanisms in the literature cannot be extended to solve the fairness problem in wireless backhaul networks with selfish nodes.

In this paper, we study the fairness problem by using game theory and propose a monetary incentive mechanism to achieve fair resource sharing for wireless backhaul networks in the presence of selfish TAPs. The fairness here is timebased, i.e., to ensure that the air time of each link used by each TAP-aggregated flow (i.e., aggregated from all of its local mobile users) is identical, and must conform with the fairness reference model proposed in [5]. The goal of a typical game theory based design is to combine the preference of each player into the outcome which satisfies the "social choice," [19] i.e., the desired outcome is that each player can optimizes his/her profit according to the rules of the game. In other words, the game is designed in such a way that a strategy that results in the social choice is the optimal or dominant strategy for each independent player. In this paper, the independent player corresponds to the TAP, and the desired outcome is achieved if the fairness in the backhaul network is realized. 
In our game, each TAP is selfish and wants to maximize its profit; each mobile user pays its associated TAP for successful data transmission. We introduce two kinds of virtual currency in the game and design an incentive mechanism by which the fairness is realized and the TAPs have no incentive to cheat and collude. We prove that our mechanism is correct and truthful, and evaluate the performance of our mechanism via ns-2 simulations. To the best of our knowledge, this is the first work which achieves fair resource sharing and acts truthfully for wireless backhaul networks in the presence of selfish TAPs.

The rest of the paper is organized as follows. In Section II, we present the network model in our design, describe the fairness reference model adopted in this paper, and introduce the virtual currency used in the game design. In Section III, we propose an incentive-based fairness mechanism in a game theory based approach. We then prove the correctness and truthfulness of this mechanism in Section IV, and evaluate the performance of the proposed mechanism via ns-2 simulations in Section V. Finally, this paper is concluded in Section VI.

\section{Network Model And Assumptions}

\section{A. Network Model}

We consider static, non-cooperative TAPs in a multi-hop wireless backhaul network. Each TAP is selfish, and its objective is to maximize its own profit. In other words, TAPs are economically rational in the sense that they will not take actions unless the profits of their actions are positive. The profit here refers to the income earned by their actions minus the cost spent in the actions. Mobile users pay the attached TAPs for sending data. The data traffic of mobile users is then forwarded through multiple intermediate TAPs to reach the gateway. We assume that data will not be exchanged between TAPs, and data from one TAP will not be split to multiple gateways at intermediate TAPs. The TAPs which have no data from local mobile users are referred to as idle TAPs; the TAPs which are not idle and with no transit data from other TAPs are referred to as border TAPs. In this paper, we do not consider the power consumption or energy efficient issues, since TAPs owned by entities should be equipped with power supplies.

Each TAP has two data queues at the network layer: one for packets in transit from other TAPs and the other for packets from its local mobile users. Data from both queues are merged at the MAC layer for further forwarding. Each TAP measures the offered load of its local traffic, i.e., the arrival rate of aggregate traffic from all its local mobile users over a predefined measurement time period. Each TAP also measures the available capacity of each link connecting to an adjacent TAP. This capacity accounts for such factors as the MAC layer overhead, the effect of wireless interference, the hidden terminal problem, and multi-rate multi-channel issues, and can be obtained via the techniques as implemented in [20,21]. In this work, we start with the case that each link is of the same capacity. Later in Section III-A, we will extend the mechanism to cover links having different capacities. The offered load and the capacity of each link incident to each TAP are announced and exchanged between TAPs periodically in a way similar to OSPF. As a result, each TAP learns the global information of the system and maintains the information in a state table, and each entry of which contains the offered load of one TAP and the capacities of all links to adjacent TAPs. Any TAPs not announcing their information for a pre-defined time period are considered inactive, and their entries will then be purged from the state table. The end-to-end throughput for each TAP-aggregated flow with fairness constraints can be computed as a function of the offered load of each TAP. The end-to-end throughput here refers to the throughput of each TAP-aggregated flow originating from where the TAP is located on the routing path to the gateway. Since the message length is relatively small, the overhead of message exchanges is modest if the measurement interval is of an order of several hundred milliseconds.

\section{B. Fairness Reference Model}

In this paper, we adopt the fairness reference model proposed by Gambiroza et al. for multi-hop wireless backhaul networks [5]. The Gambiroza's fairness model defines four objectives. First, the granularity of fairness is a TAP-aggregated flow. The egress traffic of each TAP is treated as a single aggregate flow, independent of the number of local microflows or mobile devices supported by the TAP. Second, the maximal spatial reuse must be ensured. Third, the spatial bias must be eliminated such that TAPs located more hops away from the gateway will not share disproportionately less air time than nodes less hops away. This property is essential for the deployment of multi-hop wireless backhaul architectures because mobile users in different locations should not suffer distance-dependent performance penalty. Finally, we use air time rather than throughput as the network resource to be shared fairly so as to avoid the IEEE 802.11 performance anomaly reported in [22]. Specifically, the throughput-based allocation would lead to serious performance degradation since the station with the lowest channel quality would determine the throughputs achievable for all stations.

Note that the fairness reference model described above is used to define the fairness for TAPs destined to the same gateway. It is impractical to define fairness among TAPs destined to different gateways because each gateway may be associated with its own unique network topology, wireless resource, contention, and other resource constraints. Therefore, we focus only on the backhaul network with one gateway, and the network with more gateways can be considered as multiple backhaul networks, each with a single gateway. Please also note that the fairness mechanism proposed in [5] cannot be applied to this paper directly as it assumes TAPs are cooperative and manageable.

\section{Virtual Currency and Business Model}

Two kinds of virtual currency are defined in this work: credits and tokens ${ }^{1}$. Credits have no monetary value and can only be used in the backhaul network. Each TAP generates credits by forwarding data for the TAPs at the previous hop

\footnotetext{
${ }^{1}$ The generation and management of credits and tokens can be audited either by a special hardware or software equipped with each TAP as in [16], or by a fair third party such as the central bank through a secure connection as in [15].
} 
and consumes credits in sending data packets for its local mobile users. In this way, we can encourage TAPs to increase their stocks of credits by participating in data forwarding. The exact number of credits for sending one unit data packet is determined by the target throughput of each TAP-aggregated flow in the network, which will be described in more detail in the next section.

The other type of virtual currency is tokens. Unlike credits, tokens have real monetary value. Mobile users pay tokens to their attached TAPs for sending their data; then the TAPs cash their tokens at the central bank. Similar to the concept introduced in [15], the central bank is responsible for the transactions of tokens, and nodes (including users and TAPs) can communicate with the central bank once they have a good link connection to it. The central bank, which may be owned by network operators or service providers, can earn income from the differences in the selling and buying prices of tokens. The network operators or service providers may also own TAPs. This strategy completes the business model of our designed backhaul network. To sum up, tokens, which can be cashed at the central bank, are the major revenue for TAPs. This strategy encourages TAPs to increase their stocks of credits by forwarding data for other TAPs. Therefore, the TAPs will can earn tokens by sending data for their local mobile users.

\section{INCENTIVE-BASED MECHANISM FOR PACKET FORWARDING}

In this section, we describe our incentive-based mechanism in detail. In our mechanism, the optimal strategy for each TAP is to forward transit data for other TAPs, which will further ensure that the fairness reference model defined in [5] can be realized. In what follows, we first develop a general formulation to calculate the end-to-end throughput for each TAPaggregated flow in accordance with the fairness constraints. We then describe the operations of TAPs in our mechanism, and model the forwarding behavior as a non-cooperative game for ease of analysis. Note that the target throughput for a TAP corresponds to the end-to-end throughput for the TAP. The amount of wireless bandwidth shared for each TAP accounts for the target throughput for its local mobile users and the used bandwidth to forward transit data for other TAPs.

\section{A. Target Throughput of Each TAP}

In this paper, we provide a general formulation to obtain the target throughputs for TAPs under the fairness constraints in the backhaul network. We start with the case that all TAPs in each measurement period are backlogged, i.e., the queue of each TAP for data from its local users is always backlogged. We consider the network with $N$ TAPs and $N$ TAP-aggregated flows. Each aggregate flow $f$ traverses on a pre-determined route $R_{f}$. We assume that each wireless link $l$ on route $R_{f}$ is of the same capacity $C_{l}$. Let $t_{l}^{f}$ denote the time share for flow $f$ on link $l, l \in R_{f}$. To maximize spatial reuse and link utilization, the time share assigned to flow $f$ on each link of route $R_{f}$ must satisfy the flow preservation property, i.e., the time share for flow $f$ on each link outbound must equal the time share used to forward packets of flow $f$ on any of the previous links. Insufficient air time allocation will lead to resource wastage, since the data that had been transmitted on the previous links would be dropped on this link. Excessive air time allocation will also lead to resource wastage, since the link will be idle during a part of the allocated time share. Hence, we have

$$
t_{i}^{f} C_{l}=t_{j}^{f} C_{l}, \forall i, j \in R_{f}
$$

Since the capacity of each link is assumed the same, (1) can be rewritten by

$$
t_{i}^{f}=t_{j}^{f}, \forall i, j \in R_{f}
$$

To avoid spatial bias for flows traversing through different number of hops, we have

$$
t_{l_{1}^{f}}^{f}=t_{l_{1}^{g}}^{g}, \text { for all flows } f \text { and } g
$$

where $l_{1}^{f}$ denotes the first link of flow $f$ on route $R_{f}$. Finally, we must find the bottleneck link, which is defined as the link with most traffic passing through within its interference range. Let $h_{i}$ denote the number of flows that traverse link $i$, and $C L_{i}$ denote the set of contention links, which is the set of links in the interference range of link $i$. The bottleneck link is then defined as the link with the maximum value of $\sum_{l \in C L_{i}} h_{l}$. To address this bottleneck effect, (4) is used to maximize the spatial reuse given that the bottleneck link is $i$.

$$
\sum_{f=1}^{N} \sum_{l \in C L_{i}} t_{l}^{f}=1
$$

By solving (2), (3), and (4), we obtain the time share for flow $f$ at each hop as follows.

$$
t_{l}^{f}=\left[\sum_{k \in C L_{i}} h_{k}\right]^{-1}, \forall l \in R_{f} .
$$

Let $\rho_{i}^{\prime}$ denote the target throughput which satisfies the fairness reference model for flow $f$ originating from $T A P_{i}$, provided that all TAPs are backlogged. Thus, $\rho_{i}^{\prime}$ is given by

$$
\rho_{i}^{\prime}=t_{l}^{f} C_{l}
$$

Next, we consider the case that not all TAPs are backlogged in each measurement period. Each TAP measures the average offered load of the traffic from all its local mobile users every measurement time period. If the measured offered load $\lambda_{i}$ is less than $\rho_{i}^{\prime}, T A P_{i}$ is not backlogged and its target throughput $\rho_{i}$ is $\lambda_{i}$. For non-backlogged flow originating from $T A P_{i}$, the link share time $t_{l}^{f}, \forall l \in R_{T A P_{i}}$, in (2), (3) and (4) is substituted with $t_{l}^{f}=\lambda_{i} / C_{i}$ so as to distribute the extra amount of bandwidth to backlogged TAPs. Let $h c_{f}$ denote the number of links that flow $f$ traverses over the contention links for bottleneck link $i$ (i.e., $C L_{i}$ ), and $F_{s}$ be the set of non-backlogged flows in the calculation. Thus, the time share of backlogged flow $f$ is thus given by

$$
t_{l}^{f}=\frac{1-\sum_{f \in F_{s}}\left(h c_{f} \times t_{l}^{\prime}{ }^{\prime}\right)}{\sum_{k \in C L_{i}} h_{k}-\sum_{f \in F_{s}} h c_{f}}, \forall l \in R_{f}
$$


The actual target throughput of flow $f$ originating from $T A P_{i}$, denoted by $\rho_{i}$, is given by

$$
\rho_{i}=t_{l}^{f} C_{l} .
$$

This calculation may be repeated several times since the resulting target throughputs for some TAPs after receiving the extra amount of bandwidth may exceed their offered loads. Note that we will show in a later section that each TAP will announce its true state about backlogged or non-backlogged flows even if only the backlogged TAPs can share the extra bandwidth.

If links in the network are of different capacities, we can modify (1) as

$$
t_{i}^{f} C_{i}=t_{j}^{f} C_{j}, \forall i, j \in R_{f} .
$$

Let $C_{\max }$ and $C_{\min }$ denote the maximum and minimum link capacities, respectively, in the backhaul network, and flow $f_{\min C}$ be the aggregate flow from a TAP on the link with the minimum capacity to its adjacent TAPs. Thus, we have $t_{l_{1}^{f_{\text {minC }}}}^{f_{\min }}=C_{\max } t_{u} / C_{\min }$, where $t_{u}$ is the amount of time that transmits the data of flow $f_{m m C}$ on the link with the maximum capacity (from (9)). Then, we have the following equation according to (3):

$$
t_{l_{1}^{f}}^{f}=\frac{C_{\max }}{C_{\min }} t_{u}, \text { for all flows } f .
$$

To satisfy the flow preservation property for flow $f$ on its route to the gateway according to (9), we have

$$
t_{l}^{f}=\frac{C_{\max }}{C_{\min }} t_{u} \times \frac{C_{l_{1}^{f}}}{C_{l}} \text {, for all links } \in R_{f} .
$$

Therefore, we can find the bottleneck link $i$, which is the link with the maximum value of $\sum_{f \in C F_{i}} \sum_{l \in C L_{i}} t_{l}^{f}$, and solve the following equation to obtain the value of $t_{u}$.

$$
\sum_{f \in C F_{i}} \sum_{l \in C L_{i}} t_{l}^{f}=1
$$

where $C F_{i}$ is the set of flows traversing contention links $C L_{i}$. The target throughput $\rho_{i}$ of $T A P_{i}$ is then given by

$$
\rho_{i}=t_{l_{1}^{f}}^{f} C_{l_{1}^{f}} .
$$

Flows that do not traverse the contention links for the bottleneck link can share the remaining wireless bandwidth fairly, and the calculation is similar to the cases with nonbacklogged TAPs. Note that if there are different gateways in the backhaul network, an intermediate TAP may forward its transit data to different gateways. The wireless bandwidth to forward such data should be deducted from its link capacity in calculating the target throughputs of TAPs.

\section{B. Payment-Based Packet Forwarding Mechanism}

In our mechanism, each TAP generates one credit by forwarding one unit of transit data for an adjacent TAP. Credits have no monetary value and can only be used in the network. To earn real income (i.e., the tokens paid by mobile users), TAPs must accumulate enough volume of credits to send data for their mobile users. However, the number of credits consumed in sending one unit of local data is not fixed at one but varies with the target throughput of each TAP in order to satisfy the fairness reference model. Our design philosophy is to $(i)$ control the sending rates of border TAPs in accordance with their target throughputs by assigning them an appropriate number of credits at the beginning of each measurement period; (ii) adjust the number of credits for sending one unit of local data in each measurement period, which ensures each TAP will forward all transit data for other TAPs so as to maximize the income by generating enough number of credits for sending its local data; (iii) pay tokens to the idle TAPs on the routing path so as to encourage them to actively participate in data forwarding.

Let $\alpha_{i}$ denote the number of credits that $T A P_{i}$ uses to send one unit of local data, and $S T_{i}$ be the set of TAPs whose traffic flows traverse over $T A P_{i}$. Thus,

$$
\alpha_{i}=\frac{\sum_{j \in S T_{i}} \rho_{j}}{\rho_{i}} .
$$

$\alpha_{i}$ is designed to encourage $T A P_{i}$ to forward transit data for other TAPs, and to restrict the sending rate of $T A P_{i}$ so that it will conform with the target throughput $\rho_{i}$. Border TAPs cannot generate credits by forwarding transit data since no transit flows will traverse through them. Thus, their values of $\alpha_{i}$ are initialized to 1 and $\rho_{i}$ credits are generated every measurement time period. In this way, border TAPs can send their data at a rate of $\rho_{i}$; the next hop TAPs can generate credits by forwarding these data and the generated credits can then be spent in sending data for their mobile users.

At the beginning of each measurement period, each TAP measures the offered load of its mobile users and exchanges this information with other TAPs. Each $T A P_{i}$ then determines the target throughputs of TAPs in the network as in (6), (8) or (13), and the value of $\alpha_{i}$ can be determined accordingly. Note that the number of credits consumed by each source TAP in sending one unit of local data (i.e., $\alpha_{i}$ ) is not equal to the sum of the credits generated by the TAPs that forward the data on the routing path to the gateway. We do not need to ensure the balance between the consumption and generation of credits in the system since credits have no monetary value in our mechanism. Moreover, credits cannot be rolled over to the subsequent periods. As a result, TAPs cannot send more bursty traffic than their predetermined limits and thus they have no incentive to cheat. The truthfulness analysis of this mechanism will be presented in Section IV.

Next, we discuss that idle TAPs may have no incentive to forward transit data due to the lack of local data. Let $\mu(\mu>0)$ denote the number of tokens each mobile user pays for sending one unit of data to the attached TAP, and $\eta(\eta>0)$, the total number of tokens each source TAP pays to all idle TAPs on the routing path for sending one unit of data. Note that $T A P_{i}$ obtains $\mu$ tokens once it has successfully transmitted one unit of data even though the TAPs between $T A P_{i}$ and the gateway do not provide forwarding service. Mobile users may be willing to pay tokens to their attached TAPs only when their data frames can reach the gateway. We will show soon that with our mechanism designed in this section, each TAP will provide forwarding service such that data from mobile users 


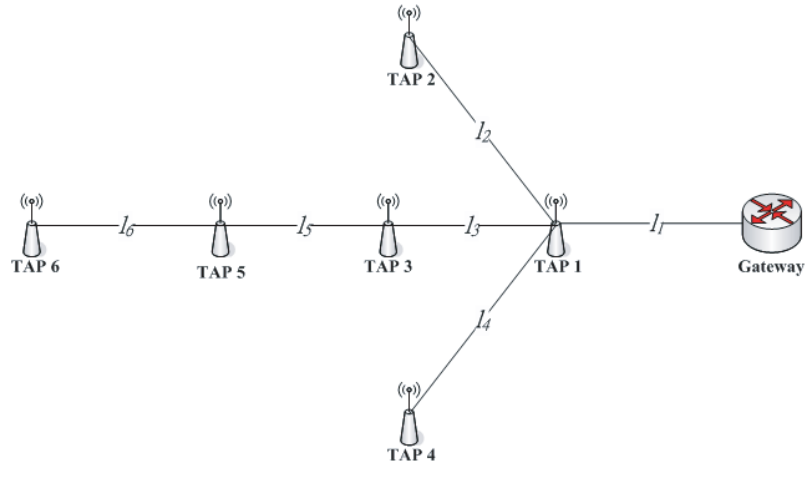

Fig. 1. An illustration of bottleneck links.

can reach the gateway successfully through multi-hop TAPs. The value of $\mu$ should be larger than $\eta$ such that the source TAPs will have an incentive to send the data. Moreover, to eliminate hop-count dependent unfairness, each source TAP needs to pay a total of $\eta$ to the idle TAPs located on its routing path, and similarly, each mobile user needs to pay the same number of tokens for sending one unit of data to its TAP regardless of the number of hops they are away from the gateway. On the contrary, the income earned by each idle TAP for forwarding one unit of transit data can be different since idle TAPs will have incentive to provide forwarding service so long as their profits are positive. Let $k_{i}$ denote the number of idle TAPs on the path from the source $T A P i$ to the gateway. Each idle $T A P i$ earns $\eta / k_{i}$ tokens by forwarding one unit of transit data. Here, the number of tokens spent by each source TAP is equal to the total number of tokens earned by the idle TAPs on the path to the gateway since tokens have monetary value. Note that the value of $\eta$ can be a small positive value close to zero or $\mu>>\eta$. Such design can ensure that the payments from their mobile users are more than the income paid by other source TAPs. This setting prevents nonidle TAPs from cheating (i.e., by announcing themselves as idle TAPs), thus ensuring that each TAP will obtain the target throughput and the fairness will be achieved.

Consider the network shown in Fig. 1 for example. Assume that the capacity of each link is $C$. The carrier sense range of the TAP is twice the transmission range so that the TAPs located two hops away from a TAP are in the carrier sense range of each TAP but not in the transmission range of the TAP. Thus link $l_{3}$ is the bottleneck link of this network since its interference range covers all links. Thus, $C L_{l_{3}}$ denotes the set of links $l_{1}, l_{2}, l_{3}, l_{4}, l_{5}$ and $l_{6}$, and $\rho_{i}^{\prime}$ for each $T A P_{i}$ is $C / 14, i=1, \ldots, 6$. Now, if the offered load of $T A P_{6}$ in Fig. 1 is only $C / 20$, which is less than its target throughput $C / 14$ when it is backlogged, $\rho_{6}$ is $C / 20$, and the air time used to transmit data for $T A P_{6}$ on links $l_{1}, l_{3}, l_{5}$ and $l_{6}$ is each $1 / 20$, and $h c_{T A P_{6}}$ is 4 . Therefore, the air time shared by the remaining contention flows is $16 / 20$ (i.e., $1-4 \times 1 / 20$ ), and the time share for each TAP, except for $T A P_{6}$, is $16 / 200$ (i.e., 16/20/(14 - 4)) according to (7). Consequently, their target throughputs are each $16 C / 200$. This leads to the fact that $T A P_{6}$ generates $C / 20$ credits, both $T A P_{2}$ and $T A P_{4}$ generate $16 C / 200$ credits in each measurement period, and the values of $\alpha_{5}$ and $\alpha_{3}$ are 10/16 (i.e., $(C / 20) /(16 C / 200)$ ) and $26 / 16$ (i.e., $(C / 20+16 C / 200) /(16 C / 200))$, respectively, according to (14). If both $T A P_{1}$ and $T A P_{3}$ are idle TAPs in this time period, all the other TAPs must each pay $\eta$ tokens for sending one unit of data, and $T A P_{3}$ and $T A P_{1}$ will obtain $\eta$ (i.e. $\eta / 2+\eta / 2$ from $T A P_{5}$ and $T A P_{6}$ ) and $3 \eta$ (i.e., $\eta / 2+$ $\eta / 2+\eta+\eta)$, respectively, tokens for forwarding one unit of transit data.

\section{Game Modeling}

Based on the discussion above, we can model the behavior of data forwarding in wireless backhaul networks as a oneround non-cooperative game since the credits cannot be rolled over to the subsequent time periods and the satisfaction of the fairness is checked in conformance with the target throughput in each measurement period.

Player There are $N$ players in the game, denoted by $T A P_{1}, T A P_{2}, \ldots, T A P_{N}$. All players are selfish and economically rational. They all attempt to maximize their individual profits, and take actions only when their profits are positive.

Players' Information The TAP knows the network topology and the offered loads of the other TAPs in the network. Thus, it can determine the target throughputs of all TAPs according to the fairness constraints and the corresponding values of $\alpha$.

Strategy Each TAP has two possible strategies: forwarding or not forwarding data for other TAPs.

Cost of Action The cost of each action mainly comes from sharing the link capacity. Since each TAP has a power supply, the cost of forwarding packets with respect to energy consumption is not considered in the game.

Payment $\left(P_{i}\right)$ If there are no idle TAPs on the path from the other source $T A P i$ to the gateway, the source $T A P_{i}$ will not pay for service availability but only consume credits; otherwise, the source TAPi must pay $\eta$ tokens per unit of data to the idle TAPs. Therefore, $P_{i}$ of $T A P_{i}$ is given by

$$
P_{i}= \begin{cases}0, & \text { if there are no idle TAPs from } T A P_{i} \\ \eta \rho_{i}, & \text { otherwise }\end{cases}
$$

Income $\left(I_{i}\right)$. The gain of non-idle $T A P_{i}$ in the system is $\mu \rho_{i}$ if the target throughput $\rho_{i}$ of $T A P_{i}$ can be achieved; otherwise, the income of $T A P_{i}$ due to forwarding transit data for other TAPs is $\sum_{j \in B} \frac{\eta \rho_{i}}{k_{j}}$, where $B$ is the set of TAPs whose routing paths traverse over $T A P_{i}$, and $k_{j}$ is the number of idle TAPs, including $T A P_{i}$, on the path from the source $T A P_{j}$ to the gateway. Thus,

$$
I_{i}= \begin{cases}\mu \rho_{i}, & \text { if } T A P_{i} \text { is not an idle TAP } \\ \sum_{j \in B} \frac{\eta \rho_{i}}{k_{j}}, & \text { otherwise. }\end{cases}
$$

Profit $\left(W_{i}\right)$. The profit of $T A P_{i}$ is the result of the received income minus the payment of the action in each measurement period, which is given by

$$
W_{i}=I_{i}-P_{i} .
$$

Note that we do not consider the regular and fixed expense for each TAP (such as leased line cost, operation and 
maintenance costs) in this work because each player wants to maximize its profit to cover these costs in the game. Instead, we consider the profit of each TAP in each measurement period. The network operators or service providers need to ensure that the profits throughout the operation period are more than these fixed costs by adjusting the values of $\mu$ and $\eta$ to ensure the soundness of the business model.

\section{ANALYSIS}

In this section, we prove fairness and truthfulness achieved by the proposed mechanism in the presence of selfish TAPs. We first show that $(i)$ forwarding data for other TAPs is the optimal strategy for all TAPs to maximize their expected profits if the values of parameters $\mu$ and $\eta$ are properly chosen in the designed mechanism, and (ii) the fairness reference model can be enforced in accordance with our mechanism. Then, we prove truthfulness by showing that each TAP in our mechanism will honestly announce the offered load of its local mobile users to the other TAPs in the network.

We first show that the social choice can be achieved in our mechanism. To prove this theorem, we have the following lemmas.

Lemma 1: Each intermediate TAP is willing to forward data for other TAPs if $\mu>\eta$ and $\eta>0$.

Proof: There are two cases to discuss.

Case 1: $T A P_{i}$ is not idle. This means that $T A P_{i}$ has data from mobile users to send. The profit of sending one unit of local data (i.e., $\mu$, or $\mu-\eta$ if there are idle TAPs between $T A P_{i}$ and the gateway) is positive if $\mu>\eta$. Note that $T A P_{i}$ earns $\mu$ tokens once it has successfully transmitted one unit of data even if the TAPs between $T A P_{i}$ and the gateway do not provide forwarding service according to our mechanism design in Section III-B. Moreover, $T A P_{i}$ must forward all transit data to maximize its income $\mu \rho_{i}$ since it needs an amount of $\alpha_{i}$ credits to send one unit of local data in our mechanism.

Case 2: $T A P_{i}$ is idle. As long as $\eta$ is positive, idle TAPs will gain profit by forwarding data.

Thus, each intermediate TAP is willing to forward all transit data for the other TAPs regardless of the strategies adopted by other TAPs in the network.

Lemma 2: The fairness reference model can be enforced in accordance with our mechanism.

Proof: We prove this lemma by showing that the shared throughput of $T A P_{i}$ is its target throughput $\rho_{i}$, regardless of whether $T A P_{i}$ is a border TAP.

Case 1: $T A P_{i}$ is a border TAP. Since $T A P_{i}$ is a border TAP, it will generate $\rho_{i}$ credits at the beginning of each measurement period. Thus, it can send the data of mobile users at a rate of $\rho_{i}$ because its value of $\alpha_{i}$ is 1 . Consequently, it can obtain this target throughput thanks to each of the intermediate TAPs being willing to forward data (from Lemma 1), and Eq. (4) or (12) is satisfied.

Case 2: $T A P_{i}$ is an intermediate TAP. Since $T A P_{i}$ will forward data for the other TAPs at previous hops according to Lemma 1, the aggregate transit data rate of $T A P_{i}$ is $\sum_{j \in S T_{i}} \rho_{j}$. As a result, $T A P_{i}$ can get $\sum_{j \in S T_{i}} \rho_{j}$ credits by forwarding data, and then can send local data at a rate of $\rho_{i}$ since we control the forwarding/sending ratio as $\alpha_{i}=$ $\sum_{j \in S T_{i}} \rho_{j} / \rho_{i}$, according to (14). Thus, $T A P_{i}$ can get its target throughput $\rho_{i}$ with our mechanism.

Theorem 1: The outcome that each independent TAP optimizes its profits according to the rules in the proposed mechanism is the desired outcome if $\mu>\eta$ and $\eta>0$.

Proof: Lemma 1 shows that the optimal strategy for each TAP is to provide forwarding service regardless of the strategies of other TAPs in the network. Thus, there is one and only one Nash equilibrium in our game, in which the strategy of each TAP is to forward transit data. Moreover, from Lemma 2, we show that the fairness model can be enforced if Lemma 1 holds. This means that the fairness model will be realized when our designed game is in Nash equilibrium. Therefore, the outcome of the game played by non-cooperative TAPs according the rules defined in our mechanism is our desired outcome.

Theorem 2: Each TAP will announce its true offered load from its mobile users if the value of $\eta$ is a positive value close to 0 or $\mu>>\eta$.

Proof: TAPs may cheat in any of four ways in the designed mechanism: $(i)$ announce a lower offered rate; $(i i)$ announce a higher offered rate; (iii) announce itself as a non-idle TAP; (iv) announce itself as an idle TAP. We show that each TAP in the backhaul network will not have an incentive to cheat.

Case 1: $T A P_{i}$ announces a lower rate. This will lead to a higher value of $\alpha_{i}$ according to (14), and so that $T A P_{i}$ can send the less amount of local data by forwarding the same value of transit data if $T A P_{i}$ is not a border TAP. Otherwise, if $T A P_{i}$ is a border TAP, the announcement of a lower rate will lead to a lower target throughput for $T A P_{i}$, and so that less credit can be generated in each measurement period. Consequently, $T A P_{i}$ will get less target throughput and have less profit paid by its mobile users than when it announces a correct offered rate. Therefore, $T A P_{i}$ has no incentive to cheat.

Case 2: $T A P_{i}$ announces a higher rate. If $T A P_{i}$ cheats and announces a higher rate than its real offered rate, the value of $\alpha_{i}$ will be smaller and the generated credits of $T A P_{i}$ can be used to send more local data. The higher the offered rate announced by a border TAP, the more the credits generated for itself. However, since $T A P_{i}$ does not have such local data to send in this time period, and the credits cannot be rolled over to the subsequent time periods in our mechanism, the shared throughput of $T A P_{i}$ remains the same. Thus, $T A P_{i}$ has no incentive to cheat.

Case 3: idle $T A P_{i}$ cheats and announces itself as a non-idle TAP. Since idle TAPs can earn $\eta / k_{i}$ tokens by forwarding one unit of data, $T A P_{i}$ will not announce itself as a non-idle TAP since it would not get any tokens from its mobile users.

Case 4: non-idle $T A P_{i}$ cheats and announces itself as an idle TAP. $T A P_{i}$ has no incentive to cheat since $\mu \rho_{i}>$ $\sum_{j \in B} \eta \rho_{j} / k_{j}$ in our mechanism if the value of $\eta$ is a positive value close to 0 or $\mu>>\eta$.

Thus, the profit of TAPs will not increase if they cheat in any one of the four cases. Moreover, collusion is helpless in our mechanism. Therefore, our mechanism is truthful. 


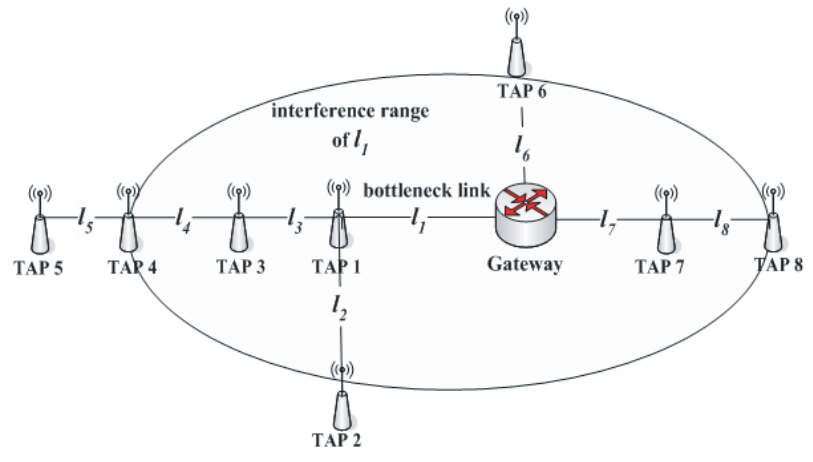

Fig. 2. Topology for the simulations.

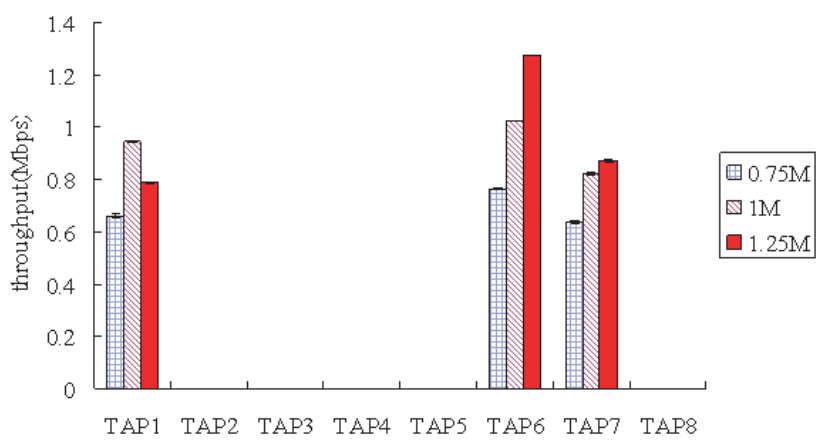

Fig. 3. Throughput performance of non-cooperative TAPs with different traffic loads.

\section{Performance Evaluation}

In this section, we evaluate the performance of the proposed mechanisms via ns-2 simulations. The wireless link rate is set to $11 \mathrm{Mbps}$ and each traffic flow is generated as CBR UDP traffic with packet size, including the IP header, fixed at 1500 bytes. The MAC protocol used in these simulations is IEEE 802.11 DCF with RTS/CTS. Fig. 2 shows the simulation topology, in which TAPs located two hops away are in the carrier sense range, but not in the transmission range. The results shown in Figs. 3-5 are plotted with $95 \%$ confidence intervals.

We first demonstrate the effect of 802.11 DCF in multi-hop wireless backhaul network when TAPs are non-cooperative. Fig. 3 shows the end-to-end throughputs of TAPs with different traffic loads (i.e., $0.75 \mathrm{Mbps}$, $1 \mathrm{Mbps}$ and $1.25 \mathrm{Mbps}$ ). We can see that if TAPs are non-cooperative, the TAPs directly connected to the gateway, such as $T A P_{1}, T A P_{6}$ and $T A P_{7}$, consumes all wireless bandwidth and the other TAPs suffer from starvation. Moreover, the wireless bandwidth contended by all TAPs other than $T A P_{1}, T A P_{6}$ and $T A P_{7}$ is wasted since the next hop TAPs will not provide forwarding service.

Then, we show the unfairness of 802.11 DCF for cooperative TAPs without our mechanism. Fig. 4 shows the simulation results with different offered rates. We can see that $(i)$ the TAPs located more hops away from the gateway obtain less end-to-end throughputs than those less hops away, resulting in spatial bias in the network; (ii) the degree of bias increases when the offered rates of TAPs increase because both transit

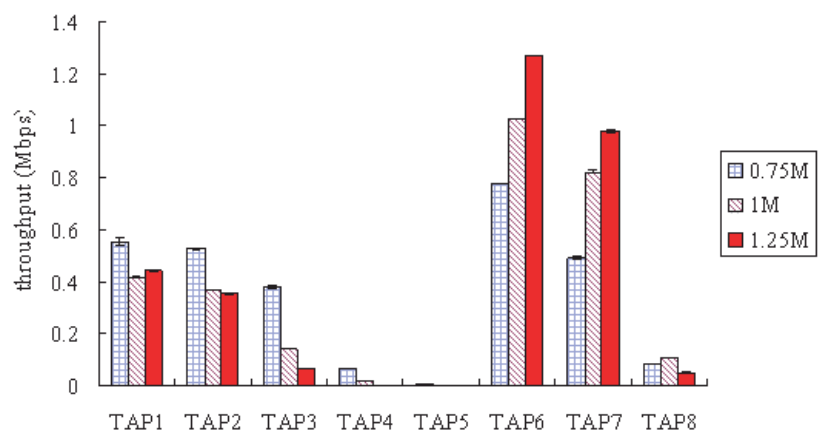

Fig. 4. Throughput performance with cooperative TAPs.

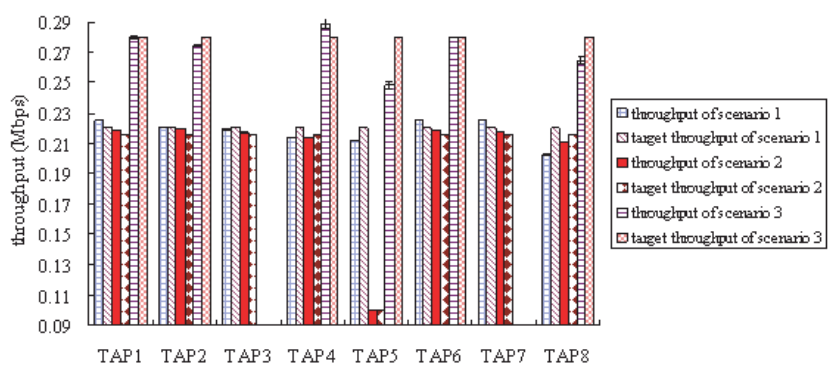

Fig. 5. Throughput performance of our mechanism.

and local data are put into the same queues, thus increasing the frame dropping rates of the data from other TAPs when the arrival rates of mobile users increase; (iii) although $T A P_{3}$ and $T A P_{2}$ are two hops away from the gateway, $T A P_{3}$ gets less end-to-end throughput because more neighbors contend for the wireless channel.

Next, we demonstrate the effect of our proposed mechanism. In our simulation, the available link bandwidth is about 3.3 Mbps, accounting for the network topology, the contention among nodes in the network and the packet size. Both $l_{1}$ and $l_{3}$ can be selected as the bottleneck links in this scenario since they both have the largest value of $\sum_{l \in C L_{i}} h_{l}$. In this simulation, we choose $l_{1}$ as the bottleneck link and the contention links of link $l_{1}$ are shown in Fig. 2. We consider three scenarios in this simulation. In the first scenario, all TAPs in the network are backlogged, and thus the target throughput of each TAP is 220kbps (i.e., 3300/15) according to our mechanism. The offered of $T A P_{5}$ drops to $100 \mathrm{kbps}$ in the second scenario and the target throughput for each of the other TAPs is about 250kbps (i.e., 3000/12). In the last scenario, $T A P_{3}$ and $T A P_{7}$ are idle. Therefore, the target throughputs for the other TAPs are each $275 \mathrm{kbps}$. Fig. 5 shows the simulation results for the simulated throughputs and the target throughputs for TAPs in different scenarios. We can see that our proposed mechanism works well even when there are idle and non-backlogged TAPs.

\section{CONCLUSION}

In this paper, we study the fairness problem in multihop wireless backhaul networks with selfish Transit Access 
Points. In our mechanism, TAPs in the backhaul network have incentives to forward data for the other TAPs and fairness can be achieved in the backhaul network. We also validate the correctness of our proposed mechanism.

In the future, we will study backhaul TAPs with different weights since some TAPs may be willing to pay more for better service. We will also consider the downlink traffic in the backhaul network, and design an efficient algorithm to calculate the target throughput for each TAP when some TAPs in the network are not backlogged.

\section{ACKNOWLEDGMENT}

This work was supported in part by National Science Council (NSC), Taiwan, under a Center Excellence Grant NSC95-2752-E-002-006-PAE, and in part by NSC under Grant Number NSC95-2221-E-001-018-MY3.

\section{REFERENCES}

[1] W. P. Atikom, P. Krishnamurthy, and S. Banerjee, "Distributed mechanisms for quality of service in wireless LANs," IEEE Trans. Wireless Commun, vol. 10, no. 3, pp. 26-34, June 2003.

[2] J.F. Lee, W. Liao, and M. C. Chen, "Inter-frame space (IFS) based distributed fair queuing in IEEE 802.11 WLANs," IEEE Trans. Veh. Technol., Mar. 2007, pp. 1366-1373.

[3] J.F. Lee, W. Liao, and M. C. Chen, "A differentiated service model for the enhanced distributed channel access (EDCA) of IEEE 802.11e WLANs," ACM/Springer Mobile Networks Applications (MONET) Special Issue Wireless Broadband Access, vol. 12, no. 1, pp. 69-77, Feb. 2007.

[4] D. Qiao and K. G. Shin, "Achieving efficient channel utilization and weighted fairness for data communications in IEEE 802.11 WLAN under the DCF," in Proc. IWQoS, 2002, pp. 227-236.

[5] V. Gambiroza, B. Sadeghi, and E. W. Knightly, "End-to-end performance and fairness in multihop wireless backhaul networks," in Proc. IEEE MOBICOM, 2004, pp. 287-301.

[6] J. Jun and M. L. Sichitiu, "Fairness and QoS in multihop wireless networks," in Proc. IEEE VTC, 2003, pp. 2936-2940.

[7] K. Sundaresan, H. Y. Hsieh, and R. Sivakumar, "IEEE 802.11 over multi-hop wireless networks: problems and new perspectives," Ad Hoc Networks, 2004, pp. 109-132.

[8] X. Wang and K. Kar, "Cross-layer rate control for end-to-end proportional fairness in wireless networks with random access," in Proc. MOBIHOC, 2005, pp. 157-168.

[9] S. Buchegger and J. Le Boudec. "Cooperative routing in mobile ad-hoc networks: Current efforts against malice and selfishness," Lecture Notes in Proc. Informatics, Mobile Internet Workshop, Informatik, 2002.

[10] S. Buchegger and J. Y. Le Boudec. "Performance analysis of the CONFIDANT protocol: Cooperation of nodes- fairness in dynamic adhoc networks," in Proc. MOBIHOC, 2002, pp. 226-236.

[11] P. Michiardi and R. Molva. "Core: A collaborative reputation mechanism to enforce node cooperation in mobile ad hoc networks," Commun. Multimedia Security, pp. 107-121, 2002.

[12] P. Resnick, K. Kuwabara, R. Zeckhauser, and E. Friedman, "Reputation systems," Commun. ACM, vol. 43, no. 12, pp. 45-48, 2000.

[13] S. Marti, T. Giuli, K. Lai, and M. Baker, "Mitigating routing misbehavior in mobile ad hoc networks," in Proc. 6th International Conf. Mobile Computing Networking, 2000.

[14] S. Buchegger and J.-Y. L. Boudec, "Nodes bearing grudges: Towards routing security, fairness, and robustness in mobile ad hoc networks," in Proc. 10th Euromicro Workshop Parallel, Distributed Network-Based Processing, 2002, 403-410.

[15] S. Zhong, Y. Yang, and J. Chen. "Sprite: A simple, cheat-proof, creditbased system for mobile ad hoc networks," in Proc. INFOCOM, 2003.

[16] L. Buttyan and J. Hubaux, "Enforcing service availability in mobile adhoc WANs," in Proc. MOBIHOC, 2000, pp. 1987-1997.
[17] M. Jakobsson, J. Hubaux, and L. Buttyan, "A micro-payment scheme encouraging collaboration in multi-hop cellular networks," in Proc. Financial Crypto, 2003, pp. 15-33.

[18] L. Anderegg and S. Eidenbenz, "Ad hoc-VCG: A truthful and costefficient routing protocol for mobile ad hoc networks with selfish agents," in Proc. MOBICOM, 2003, pp. 245-259.

[19] N. Nisan and A. Ronen, "Algorithmic mechanism design," Games Economic Behavior, vol. 35, pp. 166-196, 2001.

[20] M. Carvalho and J. J. Garcia-Luna-Aceves, "A scalable model for channel access protocols in multihop ad hoc networks," in Proc. MOBICOM, 2004, pp. 330-344.

[21] Y. Yang, J. Wang, and R. Kravets, "Achievable bandwidth prediction in multihop wireless networks," Technical Report, University of Illinois at Urbana-Champaign, Urbana, IL, 2003.

[22] M. Heusse, F. Rousseau, G. Berger-Sabbatel, and A. Duda, "Performance anomaly of 802.11b," in Proc. INFOCOM, 2003, pp. 836-843.

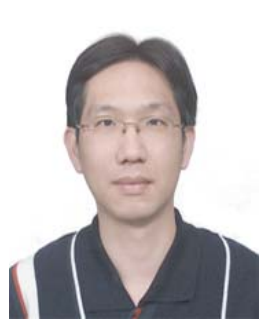

JengFarn Lee received the B.S. and M.S. degrees in the Department of Information Management from National Taiwan University, Taiwan, in 1998 and 2000, respectively, and the $\mathrm{Ph}$. D degree in the Department of Electrical Engineering from National Taiwan University, Taiwan, in 2007 . He is a postdoctoral fellow in the Institute of Information Science, Academia Sinica, Taiwan. His current research interests include QoS networking, scheduling, and wireless access networks.

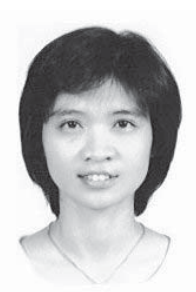

Wanjiun Liao received the BS and MS degrees from National Chiao Tung University, Taiwan, in 1990 and 1992, respectively, and the Ph.D. degree in Electrical Engineering from the University of Southern California, Los Angeles, California, USA, in 1997. She joined the Department of Electrical Engineering, National Taiwan University (NTU), Taipei, Taiwan, as an Assistant Professor in 1997. Since August 2005, she has been a full professor. Her research interests include wireless networks, multimedia networks, and broadband access net-

works.

Dr. Liao is actively involved in the international research community, and has served on the program committees of many international conferences. She is currently an Associate Editor of IEEE TRANSACTIONS ON WIRELESS Communications and the IEEE Transactions on Multimedia. Dr. Liao has received many research awards. Two papers she co-authored with her students received the Best Student Paper Award at the First IEEE International Conference on Multimedia and Expo (ICME) in 2000, and the Best Paper Award at the First International Conference on Communication, Circuits and Systems (ICCCAS) in 2002. Dr. Liao was elected as one of Ten Outstanding Young Women in Taiwan in 2000 and was the recipient of a Distinguished Research Award honored by National Science Council in Taiwan in 2006.

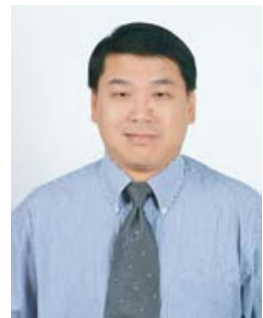

Meng Chang Chen received the B.S. and M.S. degrees in Computer Science from National ChiaoTung University, Taiwan, in 1979 and 1981, respectively, and the Ph.D. degree in Computer Science from the University of California, Los Angeles, in 1989. He joined AT\&T Bell Labs in 1989 as Member of Technical Staff and led several projects in the area of data quality of distributed databases for mission critical systems. He was an Associate Professor at Department of Information Management, National Sun Yat-Sen University, Taiwan, from 1992 to 1993 Since then he has been with Institute of Information Science, Academia Sinica, Taiwan. He has held an Associate Research Fellowship since July 1996, and assumed the responsibility of Deputy Director from August 1999 to July 2002. His current research interests include QoS networking, wireless network, multimedia systems and transmissions, operating systems, and data and knowledge engineering. 